Pacific Journal of Mathematics

BINOMIAL BEHAVIOR OF BETTI NUMBERS FOR MODULES
OF FINITE LENGTH

Edward Graham Evans, JR. AND Phillip Alan Griffin 


\title{
BINOMIAL BEHAVIOR OF BETTI NUMBERS FOR MODULES OF FINITE LENGTH
}

\section{E. G. Evans ANd Phillip Griffith}

\begin{abstract}
The problem which we address in this article is that of providing bounds for the Betti numbers of modules $M$ of finite length over a regular local ring $(R, \underline{m}, \underline{k})$ of dimension $n$. The conjectured lower bound is that the $i$ th Betti number $\beta_{i}(M)$, or $\beta_{i}^{R}(M)$ if we wish to call attention to the ring $R$, is at least $\left(\begin{array}{l}n \\ i\end{array}\right)$. We establish this inequality for finite length modules of monomial type (see definition below).
\end{abstract}

The above conjecture is implicit in a problem of Horrocks as reported by Hartshorne [5, Problem 24]. The question has also been studied by Herzog and Kuhl [6] in a slightly more general form. We discuss it briefly in our monograph [4, pp. 59-61]. Recall that $\beta_{i}(M)=$ $\operatorname{dim}_{\underline{k}} \operatorname{Tor}_{i}^{R}(\underline{k}, M)$ is the rank of the $i$ th syzygy module in a minimal free resolution of $M$. A consequence of our syzygy theorem is that, if $R$ contains a field, then $\beta_{i}(M) \geq 2 i+1$ for $i<n-1$, for any module of projective dimension $n$. Moreover these lower bounds can be achieved if one does not require $M$ to be of finite length (see [4, Corollary 3.12]). Nevertheless when $M$ is of finite length one can use the above inequality as well as the result that the dual of the resolution of $M$ is also a resolution to see that the conjectured bound is correct for dimension of $R$ at most 4 .

Our paper is divided into two sections. In the first section we establish fairly general lower bounds on the $\beta_{i}(M)$ if $R$ is isomorphic to the power series ring $\underline{k}\left[\left[X_{1}, \ldots, X_{n}\right]\right]$. We accomplish this by passing to a subring $S=\underline{k}\left[\left[g_{1}, \ldots, g_{n}\right]\right]$ where the $g_{i}$ form a system of parameters for $R$ which annihilate $M$. In this case $M$ is isomorphic to $l(M)$, the length of $M$, copies of the residue field $\underline{k}$ as an $S$-module. One compares the $R$ and $S$-free resolutions of $M$ and concludes that $\beta_{i}^{R}(M)$ is at least $(l / D)\left(\begin{array}{c}n \\ i\end{array}\right)$, where $D$ is the rank of $R$ as an $S$-module and $l=l(M)$. Simple examples show that $l / D$ can be quite small.

In the second section we generalize some results of Herzog and Kühl [6]. Their results imply that, if $R=\underline{k}\left[\left[X_{1}, \ldots, X_{n}\right]\right]$ with $R / I$ finite length and if $I$ is generated by monomials in the $X_{i}$ such that $R / I$ has a linear resolution, then $\beta_{i}(R / I)$ is at least $\left(\begin{array}{l}n \\ i\end{array}\right)$. We refer to a module 
of finite length as being of monomial type if it is a direct sum of modules $R / I$ where each $I$ is generated by monomials. In $\S 2$ we show that if $M$ is of monomial type, then $\beta_{i}(M) \geq\left(\begin{array}{l}n \\ i\end{array}\right)$. A key observation to the proof of this result is that if $M$ is an $R=\underline{k}\left[\left[X_{1}, \ldots, X_{n}\right]\right]-$ module of monomial type then $M$ is also of monomial type as an $S=\underline{k}\left[\left[X_{1}, \ldots, X_{n-1}\right]\right]$-module.

We mention that Matsumura's book [8] and our monograph [4] are standard sources for unexplained terminology.

1. Comparison of finite free resolutions with Koszul Complexes. For ease of exposition we will assume that $(R, \underline{m}, \underline{k})$ is a complete local

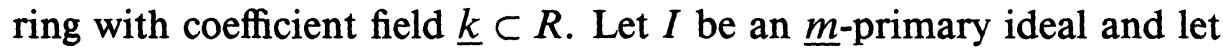
$g_{1}, \ldots, g_{n}$ be a system of parameters which is contained in $I$. Then $A=$ $k\left[\left[g_{1}, \ldots, g_{n}\right]\right]$ is a regular local subring of $R$ such that $R$ is a finitely generated $A$-module. Since the $R$ and $A$-depth of $R$ are necessarily the same, one sees that $R$ is a free $A$-module whose rank we denote by $D=[R: A]$. We now record our first result.

THEOREM 1.1. (Notation as above.) Let $\mathbf{F}$. denote a free resolution of the finite length module $M$. We assume that $M$ can be minimally generated by $\mu$ elements and that $g_{1}, \ldots, g_{n}$ is a system of parameters for $R$ which is in the annihilator of $M$. There is a homomorphism of $R /\left(g_{1}, \ldots, g_{n}\right)^{\mu}$ onto $M$ which can be lifted to a map of complexes $\phi .: \mathbf{K}^{\mu} \rightarrow \mathbf{F}$. where $\mathbf{K}$. is the Koszul complex on $g_{1}, \ldots, g_{n}$. Then, if $\phi_{i}$ is the lifting at the ith stage, we have that $\operatorname{rank} \phi_{i} \geq(l / D)\left(\begin{array}{l}n \\ i\end{array}\right)$, where $l$ is the length of $M$.

We remark that an immediate corollary of this statement is that $\beta_{i}(M) \geq(l / D)\left(\begin{array}{l}n \\ i\end{array}\right)$.

Proof. The key idea is to view the resolutions $\mathbf{K}^{\mu}$. and $\mathbf{F}$. along with the map $\phi$. of complexes over the subring $A$ as well as over $R$. Since $R$ is an $A$-free module both complexes also represent free resolutions of modules when viewed over $A$. The module $M$ viewed as an $A$ module is isomorphic to $\underline{k}^{l}$ since the maximal ideal of $A,\left(g_{1}, \ldots, g_{n}\right) A$, annihilates $M$. Hence over $A$, the resolution $\mathbf{F}$. is a direct sum $\mathbf{F}$. = $\mathbf{F}^{\prime} \otimes \mathbf{F}^{\prime \prime}$. where $\mathbf{F}^{\prime}$. is a minimal free $A$-resolution of $\underline{k}^{l}$ and $\mathbf{F}^{\prime}$. is a trivial free $A$-complex. Now looking at the complex $\mathbf{K}^{\mu}$. we note that $\mathbf{K}$. is isomorphic to $R \otimes_{A} \mathbf{K}$ ! where $\mathbf{K}^{\prime}$. is the Koszul complex for $g_{1}, \ldots, g_{n}$ over $A$. Thus when viewed over $A$ we see that $\mathbf{K}$. is isomorphic to the direct sum $\left(\mathbf{K}^{\prime}\right)^{D}$, where $D=[R: A]$. Finally considering the 
map $\phi .: \mathbf{K}^{\mu} \rightarrow \mathbf{F}$. as an $A$-map of complexes we get an induced map $\rho .: \mathbf{K}^{\mu}{ }^{\mu} \rightarrow \mathbf{F}^{\prime}$. which induces a split surjection on the zeroth homology. It follows that $\rho_{i}: K_{i}^{\mu} \rightarrow F_{i}^{\prime}$ is necessarily surjective for each $i$. Thus $\operatorname{rank}_{A} \phi_{i} \geq \operatorname{rank}_{A} \rho_{i}=l\left(\begin{array}{c}n \\ i\end{array}\right)$ for each $i$. To compute the rank of $\phi_{i}$ over $R$ we divide by $[R: A]=D$. This proves that $\operatorname{rank}_{R} \phi_{i} \geq(l / D)\left(\begin{array}{l}n \\ i\end{array}\right)$.

As we remarked above the factor $l / D$ can be quite small. In a special case we would like to pick the $g_{i}$ in such a way as to maximize $l / D$. In some cases we can relate $[R: A]$ more directly to the $g_{i}$. This is the result of the next lemma.

LEMMA 1.2. Let $\underline{k}$ be a field and let $R$ be the ring of polynomials, $R=\underline{k}\left[T_{1}, \ldots, T_{n}\right]$, which is graded by total degree. Let $g_{1}, \ldots, g_{n}$ be a homogeneous system of parameters and let $A=\underline{k}\left[g_{1}, \ldots, g_{n}\right]$. Let the degree of $g_{i}$ be $d_{i}$ for each $i$. Then $\hat{R}=\underline{k}\left[\left[T_{1}, \ldots, T_{n}\right]\right]$ is a free $\hat{A}=k\left[\left[g_{1}, \ldots, g_{n}\right]\right]$ of rank $d=\Pi d_{i}$.

Proof. Since the residue field extension from $\hat{A}$ to $\hat{R}$ is trivial we have that

$$
\begin{aligned}
\operatorname{rank}_{\hat{A}} \hat{R} & =\text { length } \hat{R} \otimes_{\hat{A}} \underline{k}=\text { length } R \otimes_{A} \underline{k} \\
& =\text { length } R /\left(g_{1}, \ldots, g_{n}\right)=\Pi d_{i} .
\end{aligned}
$$

Corollary 1.3. Let $\underline{k}$ be a field, let $R=\underline{k}\left[T_{1}, \ldots, T_{n}\right]$ and let $I$ be a graded ideal such that $R / I$ has finite length. Let $g_{1}, \ldots, g_{n}$ be a homogeneous system of parameters in $I$ with $\operatorname{deg} g_{i}=d_{i}$ and let $M$ be a finitely generated graded $R$-module which is annihilated by $I$. Then $\beta_{i}(M) \geq(l / d)\left(\begin{array}{c}n \\ i\end{array}\right)$ where $d=\Pi d_{i}$.

Proof. Obvious.

Thus one maximizes the factor $l / d$ by judiciously choosing the $g_{i}$ so as to minimize their degrees.

2. The case of modules of monomial type. We let $\underline{k}$ be a field, $S=$ $\underline{k}\left[X_{1}, \ldots, X_{d}\right]$ the ring of polynomials over $\underline{k}$ and $R=S[T]$, the ring of polynomials in one variable $T$ over $S$. We let $\underline{n}=\left(X_{1}, \ldots, X_{d}\right)$ denote the irrelevant maximal ideal of $S$ and $\underline{m}=\left(X_{1}, \ldots, X_{d}, T\right)$ the same for $R$. Three facts emerge as crucial ones in passing from $R$-modules to $S$-modules, namely:

2. (a) An $R$-module $M$ of finite length when viewed as an $S$-module has the same finite length. (We remark that a finitely generated $R$ module may fail to be finitely generated as an $S$-module and, even if it is, the number of generators may increase.) 
2. (b) An $R$-module of monomial type when viewed as an $S$-module is still of monomial type (see Lemma 2.2).

2. (c) Any $R$-module $M$ fits into a functorial $R$-short exact sequence

$$
0 \rightarrow R \otimes_{S} M \stackrel{\sigma_{M}}{\rightarrow} R \otimes_{S} M \stackrel{\varepsilon_{M}}{\rightarrow} M \rightarrow 0
$$

where $\varepsilon_{M}(r \otimes m)=r m$ and $\sigma_{M}$ is defined by

$$
\sum_{i} T^{i} \otimes m_{i} \stackrel{\sigma_{M}}{\rightarrow}-\sum_{i} T^{i}(1 \otimes T-T \otimes 1) m_{i}
$$

(see [1, p. 629] or [9, [p. 244] for details).

First we record three observations on upper bounds for Betti numbers.

Proposition 2.1. (Notation as above.) If $M$ is a graded $R$-module of finite length $l$, then for each $j \geq 1$ we have

(i) $\beta_{i}^{R}(M) \leq \beta_{j}^{S}(M)+\beta_{j-1}^{S}(M)$,

(ii) $\beta_{j}^{R}(M) \leq l\left({ }_{j}^{\operatorname{dim} R}\right)$,

(iii) $\beta_{j}^{R}(M) \leq \beta_{j}^{R}\left(M^{\prime}\right)$, where $M^{\prime}$ is the $R$-module $M$ first regarded as an $S$-module and then made into a (different) $R$-module by having $T$ act as zero.

Proof. The short exact sequence in 2.(c) above yields a long exact sequence in Tor

$$
\cdots \rightarrow \operatorname{Tor}_{j}^{R}\left(\underline{k}, R \otimes_{S} M\right) \rightarrow \operatorname{Tor}_{j}^{R}(\underline{k}, M) \rightarrow \operatorname{Tor}_{j-1}^{R}\left(\underline{k}, R \otimes_{S} M\right) \rightarrow \ldots
$$

which gives the necessary inequality on vector space dimensions to prove (i).

Part (ii) follows by induction on the dimension of $R$ using part (i) and the equality

$$
\left(\begin{array}{c}
\operatorname{dim} R \\
j
\end{array}\right)=\left(\begin{array}{c}
\operatorname{dim} S \\
j
\end{array}\right)+\left(\begin{array}{c}
\operatorname{dim} S \\
j-1
\end{array}\right)
$$

Part (iii) follows from part (i) and the fact [4; Theorem 6.21] that the inequality is an equality if $T$ acts as zero on the module in question. Then $\beta_{j}^{R}\left(M^{\prime}\right)=\beta_{j}^{S}\left(M^{\prime}\right)+\beta_{j-1}^{S}\left(M^{\prime}\right)$. But $\beta_{j}^{S}(M)=\beta_{j}^{S}\left(M^{\prime}\right)$ and $\beta_{j-1}^{S}(M)=\beta_{j-1}^{S}\left(M^{\prime}\right)$.

We remark that forming the $R$-module $M^{\prime}$ from $M$ by having $T$ act as zero has a simplifying effect on the relations. Part (iii) implies that this simplification of structure tends to enlarge the Betti numbers. The following is a simple example of this phenomena. Let $R=\underline{k}[a, b, T]$ and $I=\left(a^{2}, b^{2}, T^{2}, a b-b T\right)$. If we look at the $R$-module $R / I$ we 
see that the Betti numbers are $\beta_{0}^{R}=1, \beta_{1}^{R}=4, \beta_{2}^{R}=5$ and $\beta_{3}^{R}=2$. After passing to the ring $S=\underline{k}[a, b]$ we observe that the module $R / I$ is generated by the images of 1 and $T$. Its Betti numbers are $\beta_{0}^{S}=2$, $\beta_{1}^{S}=5, \beta_{2}^{S}=3$. Having $T$ now act as zero and using the mapping cone to resolve the new $R$-module $(R / I)^{\prime}$ (see [4, Theorem 6.21]) we get that the new Betti numbers are $\beta_{0}^{R}=2, \beta_{1}^{R}=7, \beta_{2}^{R}=8$ and $\beta_{3}^{R}=3$ which are larger as the result predicts. We remark that these calculations were done using the Bayer/Stillman program Macaulay. We have already referred to the fact that the number of generators, $\beta_{0}$, can increase in going from $\beta_{0}^{R}(M)$ to $\beta_{0}^{S}(M)$. The latter is $\beta_{0}^{R}\left(M^{\prime}\right)$. If $R$ has dimension $n$, then $\beta_{n}^{R}(M)$ is the dimension of the socle of $M$. When we pass from $R$ to $S$, the dimension of the socle can increase since we no longer require that $T m=0$ for $m$ to be in the socle. However, the socle of $M$ as an $S$-module is the socle of $M^{\prime}$. Thus we see why $\beta_{n}(M) \leq \beta_{n}\left(M^{\prime}\right)$ when $n=\operatorname{dim} R$. Our result above then establishes that the intermediate Betti numbers also do not decrease.

The previous results of this section provide crude upper bounds for the Betti numbers of finite length modules. One could ask for better bounds. However, the more delicate and significant question seems to be that of lower bounds with the Horrocks' question being a fundamental test. We will establish that bound if $M$ is a module of monomial type. First we need a lemma on the behavior of modules of monomial type. Since the question in which we are interested respects the additivity in direct sums we can pass to the cyclic case.

LEMMA 2.2. Let $I$ be an m-primary ideal of $R$ which is generated by monomials.

(a) If $f=\sum_{j=1}^{n} k_{j} f_{j}$ is a homogeneous form in $R$ with the $f_{j}$ linearly independent monomials in $X_{1}, \ldots, X_{d}, T, k_{j} \in \underline{k}$, and if $f$ is in $I$, then each $f_{j}$ is in $I$.

b) Let $p$ be the smallest integer with $T^{p+1}$ in $I$. Then $R / I$ as an $S$-module is the direct sum $\bigoplus_{j=0}^{p} S T^{j}$, where each $S T^{j}$ is a cyclic $S$-module of finite length whose annihilator is an ideal generated by monomials. Thus, if $M$ is an R-module of monomial type, it is also an $S$-module of monomial type.

(c) The $S$-summand $S T^{p}$, hereafter, denoted by $C$, of $R / I$ is an $R$-cyclic submodule of monomial type.

Proof. (a) We note that $I$ remains a graded ideal of $R$ regardless of how we assign the degrees to the variables $X_{1}, \ldots, X_{d}, T$. Consequently, by picking the degrees properly we can arrange so that each 
$f_{j}$ is homogeneous of a different degree. Since $I$ is still graded that forces each $f$ to be in $I$.

(b) We note that $1, T, \ldots, T^{p}$ generate $R / I$ as an $S$-module. It suffices to show that $1, T, \ldots, T^{p}$ are $S$-linearly independent in $R / I$. If $\sum_{j=0}^{p} s_{j} T^{j}=0$ modulo $I$ with each of the coefficients $s_{j}$ in $S$, we have that $f=\sum s_{j} T^{j}$ is in $I$. Each homogeneous component of $f$ is in $I$ since $I$ is graded. Thus each $s_{j} T^{j}$ is in $I$ as desired. To see that each cyclic $S$-module of the form $S T^{j}$ is of monomial type we observe that the $S$-annihilator of $S T^{j}$ is generated by all monomials $g$ in $S$ such that $g T^{j}$ is in $I$.

(c) The $S$-module $C$ is clearly an $R$-module since $C$ has the property that $T C=0$. Thus $C$ is a cyclic $R$-module whose annihilator is generated by the monomials of the form $m / T^{p}$ where $m$ is a monomial in $I$ which is divisible by $T^{p}$.

Our next lemma concerns the functorial exact sequence

$$
0 \rightarrow R \otimes_{S} M \rightarrow R \otimes_{S} M \rightarrow M \rightarrow 0
$$

defined at the beginning of this section.

LemMA 2.3. For an $R$-module $M$ the map $\sigma_{M}$ in the $R$-exact sequence

$$
0 \rightarrow R \otimes_{S} M \stackrel{\sigma_{M}}{\rightarrow} R \otimes_{S} M \stackrel{\varepsilon_{M}}{\rightarrow} M \rightarrow 0
$$

is equivalent to multiplication by $T$ if and only if $T M=0$.

Proof. The necessity is obvious. To see that $\sigma_{M}$ is equivalent to multiplication by $T$ it suffices to consider the generators $1 \otimes m$ where $m \in M$.

$$
\begin{aligned}
\sigma_{M}(1 \otimes m) & =-(1 \otimes T-T \otimes 1) m \\
& =-1 \otimes T m+T \otimes m=0+T(1 \otimes m)=T(1 \otimes m) .
\end{aligned}
$$

If $C$ is the $S$-module of $R / I$ generated by $T^{p}$, then we have an $R$-exact sequence

$$
0 \rightarrow C \rightarrow R / I \rightarrow R / K \rightarrow 0
$$

which is split exact as a sequence of $S$-modules. Both $C$ and $R / K$ are of monomial type over each of the rings $R$ and $S$. This short exact 
sequence yields the following $R$-commutative diagram:

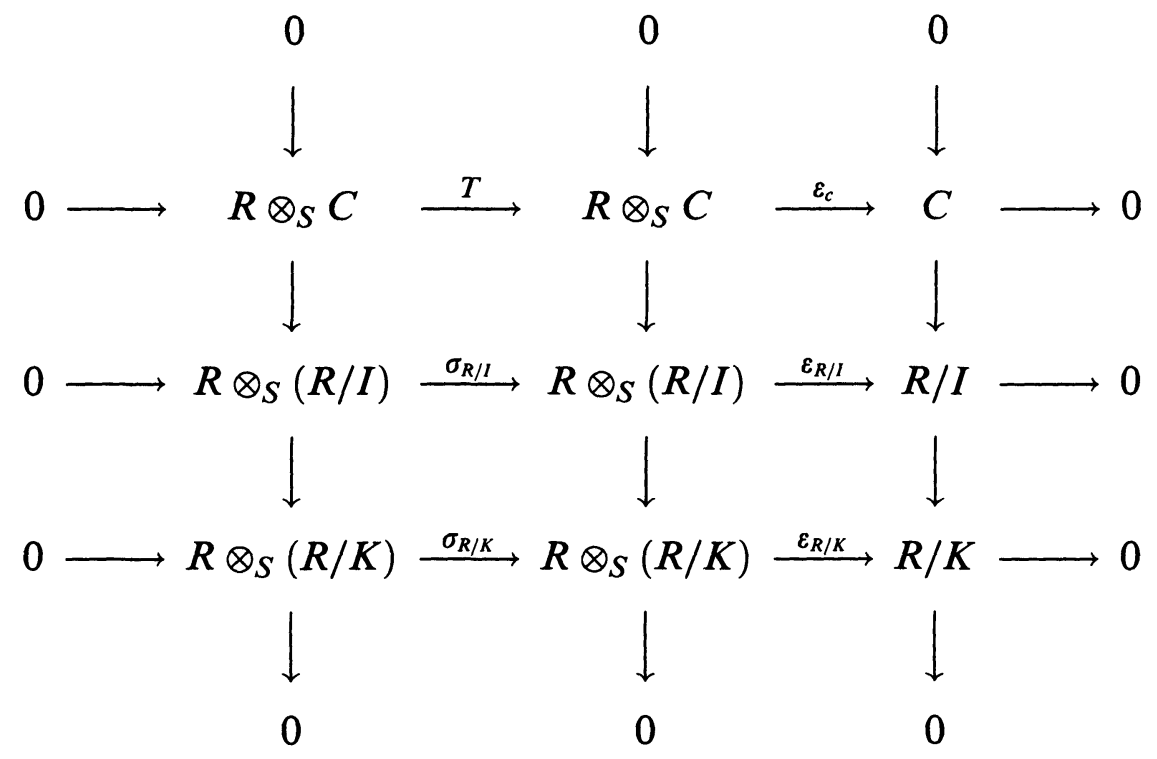

where the rows are $R$-exact, the two left hand columns are $R$-split exact and the far right hand column is $S$-split exact. Applying the functor $\operatorname{Tor}_{J}^{R}(, \underline{k})$ to the above commutative diagram we obtain a commutative diagram (see Diagram A) with $R$-exact rows and columns.

THEOREM 2.4. If $M$ is an $R$-module of monomial type and if $0 \leq$ $j \leq \operatorname{dim} R$, then

$$
\operatorname{dim}_{\underline{k}} \operatorname{Image}\left(\varepsilon_{M}^{*}: \operatorname{Tor}_{j}^{R}\left(R \otimes_{S} M, \underline{k}\right) \rightarrow \operatorname{Tor}_{j}^{R}(M, \underline{k})\right)
$$

is at least $(\underset{j}{\operatorname{dim} S})$ and

$$
\operatorname{dim}_{\underline{k}} \operatorname{Image}\left(\delta: \operatorname{Tor}_{j}^{R}(M, \underline{k}) \rightarrow \operatorname{Tor}_{j-1}^{R}\left(R \otimes_{S} M, \underline{k}\right)\right)
$$

is at least $\left(\begin{array}{c}\operatorname{dim} S \\ j-1\end{array}\right)$.

COROLlARY 2.5. If $M$ is an R-module of finite length and of monomial type, then $\beta_{j}^{R}(M) \geq\left(\operatorname{dim}_{j}^{R}\right)$ for each $j$.

The proof of the corollary is immediate from the statement of the theorem since there is a short exact sequence

$$
0 \rightarrow \text { Image } \varepsilon_{M}^{*} \rightarrow \text { Tor }_{j}^{R}(M, \underline{k}) \rightarrow \text { Image } \delta \rightarrow 0
$$

and since

$$
\left(\begin{array}{c}
\operatorname{dim} R \\
j
\end{array}\right)=\left(\begin{array}{c}
\operatorname{dim} S \\
j
\end{array}\right)+\left(\begin{array}{c}
\operatorname{dim} S \\
j-1
\end{array}\right)
$$




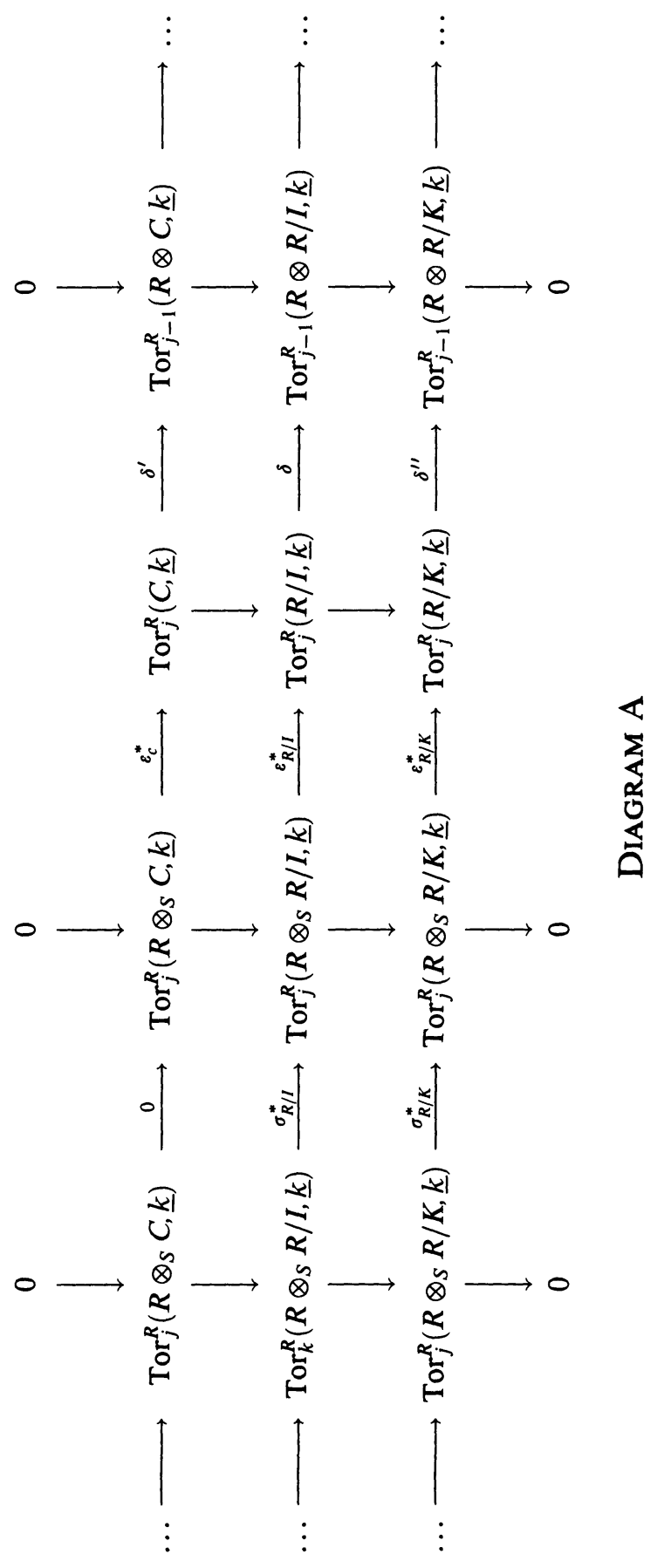


Proof of 2.4. Since the functor Tor is additive on direct sums we may pass to the case $M=R / I$ with $I$ primary to the maximal ideal and generated by monomials.

We proceed by induction on the dimension of $R$, first noting that the case $\operatorname{dim} R=1$ is obvious. After fixing the dimension of $R$ we secondly argue by induction on the smallest nonnegative integer $p$ such that $T^{p+1} \in I$. If $p=0$, then $T \in I$ and $R / I$ is a cyclic $S$-module. By Lemma $2.3 \sigma_{R / I}$ is multiplication by $T$ and thus the functorial short exact sequence

$$
0 \rightarrow R \otimes_{S} R / I \stackrel{T}{\rightarrow} R \otimes_{S} R / I \rightarrow R / I \rightarrow 0
$$

yields a short exact sequence, for $j \geq 1$,

$0 \rightarrow \operatorname{Tor}_{j}^{R}\left(R \otimes_{S} R / I, \underline{k}\right) \stackrel{\varepsilon_{R / I}^{*}}{\longrightarrow} \operatorname{Tor}_{j}^{R}(R / I, \underline{k}) \stackrel{\delta}{\rightarrow} \operatorname{Tor}_{j-1}^{R}\left(R \otimes_{S} R / I, \underline{k}\right) \rightarrow 0$.

Since $\operatorname{Tor}_{j}^{R}\left(R \otimes_{S} R / I, \underline{k}\right)$ is isomorphic to $\operatorname{Tor}_{j}^{S}(R / I, \underline{k})$, it follows by induction on the dimension of $R$ that $\operatorname{dim}_{\underline{k}} \operatorname{Tor}_{j}(R / I, \underline{k}) \geq\left(\operatorname{dim}_{j} S\right)$. Hence the images of $\varepsilon_{R / I}^{*}$ and $\delta$ have dimension at least $(\underset{j}{\operatorname{dim} S})$ and $\left(\begin{array}{c}\operatorname{dim} S \\ j-1\end{array}\right)$, respectively.

In order to argue the induction step on $p$ for $p \geq 1$, we use the short exact sequence

$$
0 \rightarrow C \rightarrow R / I \rightarrow R / K \rightarrow 0 .
$$

Now $C$ is a cyclic $S$-module of monomial type while $R / K$ is a cyclic $R$-module of monomial type with $p$ one lower.

Since the map $\operatorname{Tor}_{j}^{R}\left(R \otimes_{S} R / I, \underline{k}\right) \rightarrow \operatorname{Tor}_{j}^{R}\left(R \otimes_{S} R / K, \underline{k}\right)$ is surjective the dimension of the image of $\varepsilon_{R / I}^{*}$ is at least as large as the dimension of the image of $\varepsilon_{R / K}^{*}$ which is at least $(\underset{j}{\operatorname{dim} S})$ by induction.

Turning our attention to the connecting homomorphism $\delta$, we note that the map $\operatorname{Tor}_{j-1}^{R}\left(R \otimes_{S} C, \underline{k}\right) \rightarrow \operatorname{Tor}_{j-1}^{R}\left(R \otimes_{S} R / I, \underline{k}\right)$ is a monomorphism. The dimension of the image of $\delta$ is at least as large as that of $\delta^{\prime}$ which is at least $\left(\begin{array}{c}\operatorname{dim} S \\ j-1\end{array}\right)$ by induction.

This completes the proof of the theorem and its corollary.

\section{REFERENCES}

[1] H. Bass, Algebraic K-Theory, Benjamin Inc., New York, 1958.

[2] S. Dutta, On the canonical element conjecture, to appear.

[3] E. G. Evans and P. Griffith, The syzygy problem, Annals of Math., 114 (1981), 323-333. 
[4] - Syzygies, London Math. Soc. Lecture Note Series, 106, Cambridge University Press, Cambridge, 1985.

[5] R. Hartshorne, Algebraic vector bundles on projective spaces: a problem list, Topology 18 (1979), 117-128.

[6] J. Herzog and M. Kühl, On the Bettinumbers of finite pure and linear resolutions, Comm. Algebra, 12 (1984), 1627-1646.

[7] M. Hochster, Canonical elements in local cohomology modules and the direct summand conjecture, J. Algebra, 84 (1983), 503-553.

[8] H. Matsumura, Commutative Algebra, W. A. Benjamin Inc., New York, 1970.

[9] J. Rotman, An Introduction to Homological Algebra, Academic Press, New York, 1979.

Received October 24, 1986.

UNIVERSITY OF ILLINOIS

URBANA, IL 61801 


\title{
PACIFIC JOURNAL OF MATHEMATICS
}

\section{EDITORS}

\author{
V. S. VARADARAJAN \\ (Managing Editor) \\ University of California \\ Los Angeles, CA 90024 \\ HERBERT ClEMENS \\ University of Utah \\ Salt Lake City, UT 84112 \\ R. FINN \\ Stanford University \\ Stanford, CA 94305
}

\author{
HERMANN FLASCHKA \\ University of Arizona \\ Tucson, AZ 85721
}

RAMESh A. GANGOLLI University of Washington Seattle, WA 98195

VAUGHAN F. R. JONES University of California

Berkeley, CA 94720

\author{
ROBION KIRBY \\ University of California \\ Berkeley, CA 94720 \\ C. C. MOORE \\ University of California \\ Berkeley, CA 94720 \\ HAROLD STARK \\ University of California, San Diego \\ La Jolla, CA 92093
}

\section{ASSOCIATE EDITORS}

\author{
R. ARENS \\ E. F. BECKENBACH \\ B. H. NEUMANN \\ F. WOLF \\ K. YOSHIDA \\ (1906-1982)

\section{SUPPORTING INSTITUTIONS}

\begin{abstract}
UNIVERSITY OF ARIZONA
UNIVERSITY OF BRITISH COLUMBIA

UNIVERSITY OF CALIFORNIA

MONTANA STATE UNIVERSITY

UNIVERSITY OF NEVADA, RENO

NEW MEXICO STATE UNIVERSITY

OREGON STATE UNIVERSITY
\end{abstract} \\ CALIFORNIA INSTITUTE OF TECHNOLOGY \\ UNIVERSITY OF OREGON \\ UNIVERSITY OF SOUTHERN CALIFORNIA \\ STANFORD UNIVERSITY \\ UNIVERSITY OF HAWAII \\ UNIVERSITY OF TOKYO \\ UNIVERSITY OF UTAH \\ WASHINGTON STATE UNIVERSITY \\ UNIVERSITY OF WASHINGTON
}

The Supporting Institutions listed above contribute to the cost of publication of this Journal, but they are not owners or publishers and have no responsibility for its content or policies.

Mathematical papers intended for publication in the Pacific Journal of Mathematics should be in typed form or offset-reproduced (not dittoed), double spaced with large margins. Please do not use built up fractions in the text of the manuscript. However, you may use them in the displayed equations. Underline Greek letters in red, German in green, and script in blue. The first paragraph must be capable of being used separately as a synopsis of the entire paper. In particular it should contain no bibliographic references. Please propose a heading for the odd numbered pages of less than 35 characters. Manuscripts, in triplicate, may be sent to any one of the editors. Please classify according to the scheme of Math. Reviews, Index to Vol. 39. Supply name and address of author to whom proofs should be sent. All other communications should be addressed to the managing editor, or Elaine Barth, University of California, Los Angeles, California 90024.

There are page-charges associated with articles appearing in the Pacific Journal of Mathematics. These charges are expected to be paid by the author's University, Government Agency or Company. If the author or authors do not have access to such Institutional support these charges are waived. Single authors will receive 50 free reprints; joint authors will receive a total of 100 free reprints. Additional copies may be obtained at cost in multiples of 50 .

The Pacific Journal of Mathematics is issued monthly as of January 1966. Regular subscription rate: $\$ 190.00$ a year (5 Vols., 10 issues). Special rate: $\$ 95.00$ a year to individual members of supporting institutions.

Subscriptions, orders for numbers issued in the last three calendar years, and changes of address should be sent to Pacific Journal of Mathematics, P.O. Box 969, Carmel Valley, CA 93924, U.S.A. Old back numbers obtainable from Kraus Periodicals Co., Route 100, Millwood, NY 10546.

The Pacific Journal of Mathematics at P.O. Box 969, Carmel Valley, CA 93924 (ISSN 0030-8730) publishes 5 volumes per year. Application to mail at Second-class postage rates is pending at Carmel Valley, California, and additional mailing offices. Postmaster: send address changes to Pacific Journal of Mathematics, P.O. Box 969, Carmel Valley, CA 93924.

\section{PUBLISHED BY PACIFIC JOURNAL OF MATHEMATICS, A NON-PROFIT CORPORATION}




\section{Pacific Journal of Mathematics}

\section{Vol. 133, No. 2 \\ April, 1988}

William Charles Bauldry, Attila Mate and Paul Nevai, Asymptotics for solutions of systems of smooth recurrence equations . . . . . . . . . . 209

Ehrhard Behrends, Isomorphic Banach-Stone theorems and isomorphisms which are close to isometries ............................229

Fernanda Maria Botelho, Rotation sets of maps of the annulus .........251

Edward Graham Evans, Jr. and Phillip Alan Griffith, Binomial behavior

of Betti numbers for modules of finite length . ................. 267

Andrei Iordan, Pseudoconvex domains with peak functions at each point of

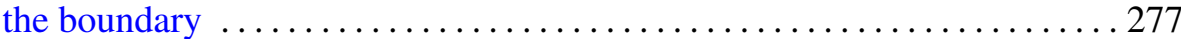

Zyun'iti Iwase, Dehn-surgery along a torus $T^{2}$-knot $\ldots \ldots \ldots \ldots \ldots \ldots . \ldots 289$

Marko Kranjc, Embedding 2-complexes in $\mathbf{R}^{4} \ldots \ldots \ldots \ldots \ldots \ldots \ldots \ldots \ldots$

Aloys Krieg, Eisenstein-series on real, complex, and quaternionic

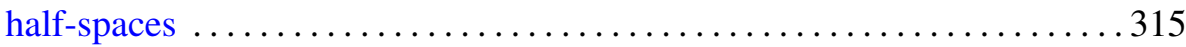

Masato Kuwata, Intersection homology of weighted projective spaces and

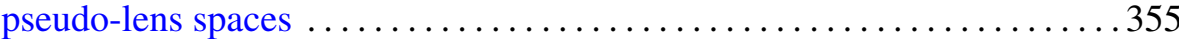

Carl Pomerance, András Sárközy and Cameron Leigh Stewart, On

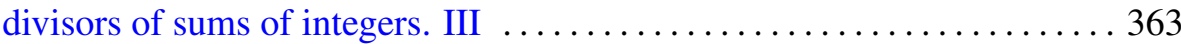

Martin Schechter, Potential estimates in Orlicz spaces ............... 381 